\title{
A numerical algorithm of parameter identification in mathematical model of tuberculosis transmission with control programs
}

\author{
S.I. Kabanikhin ${ }^{1,2}$, O.I. Krivorotko ${ }^{1,2}$, V.N. Kashtanova ${ }^{2 *}$ \\ ${ }^{1}$ Institute of Computational Mathematics and Mathematical Geophysics SB RAS, Novosibirsk, Russia \\ ${ }^{2}$ Novosibirsk State University, Novosibirsk, Russia \\ *e-mail: vikakashtanova@ya.ru
}

Key words: model of tuberculosis transmission, reconstruction of model parameters, system of ordinary differential equations, parameter identification, inverse problem, optimization approach, fast simulate annealing, gradient descent method

Motivation and Aim: The development of an individual mathematical model describing the process of the propagation of Tuberculosis (TB) infection in the population is one of the most effective methods for prediction of the epidemic spread in a particular region. Such models are described by systems of nonlinear ordinary differential equations (ODE) with the coefficients that characterize the features of population and disease spread. Consequently, it is necessary to qualitatively evaluate parameters of model (or their combinations) [1] for specification model for special population.

Methods and Algorithms: The purpose of this work is the construction and investigation of the numerical algorithm for determining the coefficients of nonlinear ODE system which describes TB transmission processes with treatment and drug resistance [2] using additional information about a special population according to statistical data for the previous few years (namely, the number of healthy, latently infected and infectious diseases individuals). The numerical algorithm is based on combination of very fast annealing and gradient approaches for minimization of least squares objective function [3]. Results and Conclusion: The results of numerical calculations show that above approach determines the set of more sensitive parameters to a particular region that differs significantly from its widely used standard values. The numerical results are analyzed and discussed.

Acknowledgments: This work is supported by the Scholarship of the President of RF No. MK-1214.2017.1. and by the grant No. 18-71-10044 of Russian Scientific Found (RScF).

\section{References}

1. Kabanikhin S.I. (2011) Inverse and Ill-Posed Problems: Theory and Applications (Berlin: de Gruyter).

2. Trauer J.M., Denholm J.T., McBryde E.S. (2014) Construction of a mathematical model for tuberculosis transmission in highly endemic regions of the Asia-pacific, Journal of Theoretical Biology, 358:74-84.

3. Banks H.T., Hu Sh., Thompson W.C. (2014) Modeling and Inverse Problems in the Presence of Uncertainty (Chapman and Hall/CRC press). 